\title{
Absence of First-order Transition and Tri-critical Point in the Dynamic Phase Diagram of a Spatially Extended Bistable System in an Oscillating Field
}

\author{
G. Korniss ${ }^{1}$, P. A. Rikvold ${ }^{2}$, and M. A. Novotny ${ }^{3}$ 月 \\ ${ }^{1}$ Department of Physics, Applied Physics, and Astronomy, \\ Rensselaer Polytechnic Institute, 110 8th Street, Troy, NY 12180-3590 \\ ${ }^{2}$ Center for Materials Research and Technology, School of Computational Science and Information Technology, \\ and Department of Physics, Florida State University, Tallahassee, Florida 32306-4350 \\ ${ }^{3}$ Department of Physics and Astronomy, Mississippi State University, \\ P.O. Drawer 5167, Mississippi State, MS 39762-5167
}

(Dated: October 22, 2018)

\begin{abstract}
It has been well established that spatially extended, bistable systems that are driven by an oscillating field exhibit a nonequilibrium dynamic phase transition (DPT). The DPT occurs when the field frequency is on the order of the inverse of an intrinsic lifetime associated with the transitions between the two stable states in a static field of the same magnitude as the amplitude of the oscillating field. The DPT is continuous and belongs to the same universality class as the equilibrium phase transition of the Ising model in zero field [G. Korniss et al., Phys. Rev. E 63, 016120 (2001); H. Fujisaka et al., Phys. Rev. E 63, 036109 (2001)]. However, it has previously been claimed that the DPT becomes discontinuous at temperatures below a tricritical point [M. Acharyya, Phys. Rev. E 59, 218 (1999)]. This claim was based on observations in dynamic Monte Carlo simulations of a multipeaked probability density for the dynamic order parameter and negative values of the fourthorder cumulant ratio. Both phenomena can be characteristic of discontinuous phase transitions. Here we use classical nucleation theory for the decay of metastable phases, together with data from large-scale dynamic Monte Carlo simulations of a two-dimensional kinetic Ising ferromagnet, to show that these observations in this case are merely finite-size effects. For sufficiently small systems and low temperatures, the continuous DPT is replaced, not by a discontinuous phase transition, but by a crossover to stochastic resonance. In the infinite-system limit the stochastic-resonance regime vanishes, and the continuous DPT should persist for all nonzero temperatures.
\end{abstract}

PACS numbers: $\quad 64.60 . \mathrm{Ht}, 75.10 . \mathrm{Hk}, 64.60 . \mathrm{Qb}, 05.40 .-\mathrm{a}$

\section{INTRODUCTION}

Metastability and hysteresis are exhibited by numerous natural and artificial systems that are driven away from thermodynamic equilibrium by an external "field." In the absence of such a field, and below some critical temperature or analogous control parameter, a large class of such systems possess two equivalent, symmetry-broken ordered phases. The external field selects one of these ordered phases as the global, stable minimum in the multidimensional freeenergy landscape. The other ordered phase becomes metastable, separated from the basin of attraction of the stable phase by a free-energy barrier. If the system is initially prepared in the metastable phase and thermal fluctuations are present, the system eventually (possibly after an extremely long time) escapes from the metastable free-energy well and approaches stable equilibrium. In the present paper we consider the response of a spatially extended, bistable system driven by an external field which is periodic in time. In particular, we focus on the finite-size effects of the periodic system response.

Ferromagnets are perhaps the most commonly known systems that exhibit metastability and hysteresis. In this paper we therefore use magnetic language, in which the order parameter is the system magnetization $m$, and its conjugate field is the external magnetic field $H$. Analogous interpretations, e.g., using the terms "polarization" and "electric field" for ferroelectric systems, "coverage" and "electrochemical potential" for adsorbate systems, etc., are straightforward [1].

The dynamic response under an oscillating external field can be viewed as a competition between two time scales: the half-period $t_{1 / 2}$ of the external field (proportional to the inverse driving frequency) and the average metastable lifetime $\langle\tau\rangle$ of the system (the mean time spent in the metastable well) after a sudden field reversal. For low driving

\footnotetext{
*Electronic address: korniss@rpi.edu

${ }^{\dagger}$ Electronic address:

‡Electronic address: man40@ra.msstate.edu
} 
frequencies, the time-dependent magnetization oscillates about zero in synchrony with the external field (symmetric dynamic phase). For high frequencies, however, the magnetization does not have time to switch sign during one half-period, and it oscillates about one or the other of its degenerate zero-field values (asymmetric dynamic phase). This symmetry breaking and the corresponding dynamic phase transition (DPT) between the symmetric and the asymmetric limit cycles of the system magnetization have attracted considerable attention over the last decade. It was first observed during numerical integration of the mean-field equation of motion for the magnetization of a ferromagnet in an oscillating field 12, 37. Since then it has been the focus of investigation in numerous Monte Carlo simulations of kinetic Ising systems [1, 4, 5, 6, 7, 8, 9, 10, 11, 12, 13, 14, further mean-field studies [5, 7, 8, 15, 16, and most recently in analytic studies of a bistable time-dependent Ginzburg-Landau (TDGL) model 117. The DPT may also have been experimentally observed in $\mathrm{Co}$ on $\mathrm{Cu}(001)$ ultrathin magnetic films [18, 19, 20] and recently in numerical studies of fully frustrated Josephson-junction arrays [21] and anisotropic Heisenberg models [22]. The results of these studies agree that there exists a genuine continuous phase transition between the symmetric and asymmetric dynamic phases, at least in some region of the parameter space spanned by temperature, field, and half-period. Finite-size scaling studies of data from dynamic Monte Carlo simulations [1, 12, 14, as well as analytic arguments 117 have demonstrated that this far-from-equilibrium phase transition belongs to the same universality class as the equilibrium Ising model in zero field. This result is consistent with previous symmetry [23] and renormalization group 24] arguments [25].

In their original paper on the DPT in a mean-field model [2], Tomé and de Oliveira reported that the continuous (second-order) phase transition observed at high temperatures appeared to change at a tricritical point (TCP) to a discontinuous (first-order) transition for low temperatures. Such a TCP was also reported in later mean-field work [5]. However, an analytical and numerical mean-field study by Zimmer 16 makes a strong case that the claims for a TCP in the mean-field case is based on a misinterpretation of effects of critical slowing-down at the DPT. Similar claims, that in some region of the dynamic phase diagram spatially extended kinetic Ising models exhibit a first-order transition and consequently have a TCP separating lines of second-order and first-order dynamic phase transitions, have also been made on the basis of dynamic Monte Carlo studies [9, 26, 27]. For recent reviews on the DPT, see Refs. [26, 27.

The purpose of the present paper is to clear up the remaining confusion about the interpretation of simulation results for the DPT in spatially extended kinetic Ising models, in particular in the low-temperature regime where a first-order transition and a TCP have been claimed to exist [9, 26, 27]. Those conclusions were essentially based on data for a single system size, and we here demonstrate how proper consideration of rather subtle finite-size effects leads to a different picture. The implication of our theoretical arguments and Monte Carlo simulations presented in this paper is that in an infinitely large system a continuous DPT should persist down to arbitrarily low temperatures. However, in any finite system for sufficiently low frequencies, the DPT gives way to a transient regime of stochastic resonance (SR) 28 at a size-dependent temperature. It is this size-dependent crossover temperature, which has previously been misinterpreted as a TCP.

The rest of this paper is organized as follows. In Sec. II we summarize the theoretical framework needed to understand the underlying metastable decay mechanisms and their consequences for the DPT. This underscores again the importance of the interplay of various time- and length scales in metastable systems [1, 10, 11, 29, 30, 31]. In Sec. [II] we extend our preliminary numerical work [32], supporting our theoretical arguments by large-scale Monte Carlo simulations of a two-dimensional kinetic Ising ferromagnet in an oscillating field. Our conclusions are summarized in Sec. IV, and derivations of analytic approximations for quantities of interest in the stochastic-resonance regime are given in Appendix A and Appendix B.

\section{METASTABLE DECAY MODES AND PERIODIC RESPONSE IN FINITE SYSTEMS}

The appropriate dynamic order parameter in the presence of an oscillating external field is the period-averaged magnetization, $Q=\frac{1}{2 t_{1 / 2}} \oint m(t) d t$ [2]. It takes a nonzero value in the asymmetric dynamic phase, while it vanishes in the symmetric phase. The transition occurs when the half-period $t_{1 / 2}$ and the underlying metastable lifetime $\langle\tau(T, H)\rangle$ become comparable. The metastable lifetime depends on the temperature $T$ and the field amplitude $H$. For sufficiently large systems (see quantitative statements below) the system escapes from the metastable phase through the nucleation of many droplets [multi-droplet (MD) regime [29, 30]]. Consequently, the time-dependent system magnetization is self-averaging. If $\langle\tau(T, H)\rangle \ll t_{1 / 2}$, the magnetization follows the external field in each half-period. The system relaxes to a symmetric limit cycle, and the order-parameter probability density $P(Q)$ is sharply peaked about $Q=0$. On the other hand, for $\langle\tau(T, H)\rangle \gg t_{1 / 2}$ the magnetization does not have enough time to switch within a single half-period, and it relaxes to an asymmetric limit cycle (with occasional switches between the two equivalent asymmetric dynamic phases). Consequently, $P(Q)$ becomes bimodal with sharp peaks near $Q= \pm 1$. This breaking of the symmetry of the limit cycle and the associated DPT have been carefully analyzed [1, 12, 14 with finite-size scaling techniques 
borrowed from equilibrium critical phenomena [33, 34]. In terms of the dimensionless half-period, $\Theta \equiv t_{1 / 2} /\langle\tau(T, H)\rangle$, the DPT occurs at a critical value $\Theta_{c} \sim \mathcal{O}(1)$. The finite-size scaling analysis of the Monte Carlo data also indicates that this far-from-equilibrium phase transition belongs to the same universality class as the equilibrium Ising model in zero field. Supporting these numerical studies, recent analytic results within a coarse-grained TDGL model 17] indicate that the behavior of the stochastic variable $Q$ is governed by the effective Hamiltonian $\mathcal{H}_{\text {eff }}=a Q^{2}+Q^{4}$, where $a \propto\left(\Theta-\Theta_{c}\right)$. According to standard arguments from the theory of critical phenomena, this result leads directly to the conclusion that the DPT belongs to the universality class of the zero-field Ising model in equilibrium [35], in agreement with the simulation results.

For any finite system, however, the metastable decay mode changes to the nucleation and growth of a single droplet at sufficiently low temperatures [single-droplet (SD) regime [29, 30]]. Due to the stochastic nature of the nucleation of a single droplet, the corresponding response in the presence of an oscillating field is different: the system exhibits [9, 11, 31] stochastic resonance 28.

The crossover from the underlying MD to SD decay can be understood by using standard nucleation theory applied to the ferromagnetic kinetic Ising model (in general dimension $d$ and with ferromagnetic coupling constant $J$ ) [29, 30]. Below the critical temperature, following a single, instantaneous field reversal, the average time between nucleation events (the nucleation time) in a system of linear size $L$ is obtained as

$$
t_{\mathrm{n}}=\left[L^{d} I(T, H)\right]^{-1},
$$

where $I(T, H)$ is the temperature- and field-dependent nucleation rate per unit volume. It can be expressed in terms of the free energy $F(T, H)$ of the critical droplet as

$$
I(T, H)=C(T, H)^{-1} e^{-F(T, H) / T},
$$

where $F(T, H)$ and the prefactor $C(T, H)$ can be obtained from nucleation theory to various degrees of approximation, depending on $T$ and $H$ [30. The temperature $T$ is given in energy units by setting the Boltzmann constant $k_{\mathrm{B}}=1$ in Eq. (2). The other characteristic time scale is the growth time $t_{\mathrm{g}}$. It is defined as the time it takes for a supercritical droplet to grow to fill half the system volume. Assuming a time-independent radial growth velocity $v(T, H)$,

$$
t_{\mathrm{g}}(L, T, H)=\frac{L}{\left[2 \Omega_{d}(T)\right]^{1 / d} v(T, H)},
$$

where $\Omega_{d}(T)$ is a dimension- and temperature-dependent shape factor (between $\pi$ and 4 for $d=2$ and between $4 \pi / 3$ and 8 for $d=3$ ] [30].

For $t_{\mathrm{n}} \ll t_{\mathrm{g}}$ many droplets nucleate while those nucleated shortly after the field reversal are still growing. In this, the MD, regime the lifetime is independent of the system size. An order-of-magnitude estimate of the metastable lifetime $\langle\tau(T, H)\rangle$ can be obtained [30] by equating the volume $R_{0}^{d}$ reached by a droplet that grows for this amount of time, $R_{0}^{d} \propto(v\langle\tau\rangle)^{d}$, with the volume inside which on average a single nucleation event occurs during the same time, $R_{0}^{d} \propto(\langle\tau\rangle I)^{-1}\left(R_{0}\right.$ can be regarded as the typical droplet separation). The result is $\langle\tau\rangle \propto\left(v^{d} I\right)^{-1 /(d+1)}$.

For $t_{\mathrm{n}} \gg t_{\mathrm{g}}$ the first droplet to nucleate eventually fills the system on its own. In this regime the lifetime depends strongly on the system size and approximately equals $t_{\mathrm{n}}$ [Eq. (1)].

The above two decay modes characterize the MD and SD regime, respectively. The crossover between the two regimes defines the dynamic spinodal (DSP) [30] and can be estimated by equating $t_{\mathrm{n}}$ and $t_{\mathrm{g}}$. (In terms of the underlying length scales, this corresponds to the situation in which the mean droplet separation $R_{0}=v\langle\tau\rangle$ becomes comparable to the system size $L$.) This yields an implicit equation for the temperature corresponding to the DSP as a function $L$ and $H$,

$$
T_{\mathrm{DSP}}=\frac{F\left(T_{\mathrm{DSP}}, H\right)}{(d+1) \ln L-\ln \left\{C\left(T_{\mathrm{DSP}}, H\right)\left[2 \Omega_{d}\left(T_{\mathrm{DSP}}\right)\right]^{1 / d} v\left(T_{\mathrm{DSP}}, H\right)\right\}} .
$$

For $d=2$, analytic approximations [36] are known for $v(T, H)$ [37], $\Omega_{2}(T)$, and $C(T, H)$ [38, 39]. The corresponding estimate for $T_{\mathrm{DSP}}$ as a function of $L$ at $H=2.0 \mathrm{~J}$, obtained by numerically solving Eq. (4), is shown as a dashed curve in Fig. 1. The resulting curve $T_{\mathrm{DSP}}(L)$ turns out to be quite insensitive for the precise values used in the approximation [36]. In the large-system limit $T_{\mathrm{DSP}}$ approaches zero logarithmically with increasing $L$. The existence of the DSP implies that in the presence of an oscillating field, reducing $T$ at fixed half-period $t_{1 / 2}$ and field amplitude $H$ results in drastically different behaviors for "small" and "large" systems.

Upon reducing the temperature for sufficiently large $L$, the $L$-independent metastable lifetime $\langle\tau(T, H)\rangle$ becomes comparable to the fixed half-period $t_{1 / 2}$ at a temperature above the one at which the underlying decay mode would cross over to the SD mode. The horizontal line in Fig. 1 corresponds to the temperature where $\langle\tau(T, H)\rangle \approx t_{1 / 2}=$ 


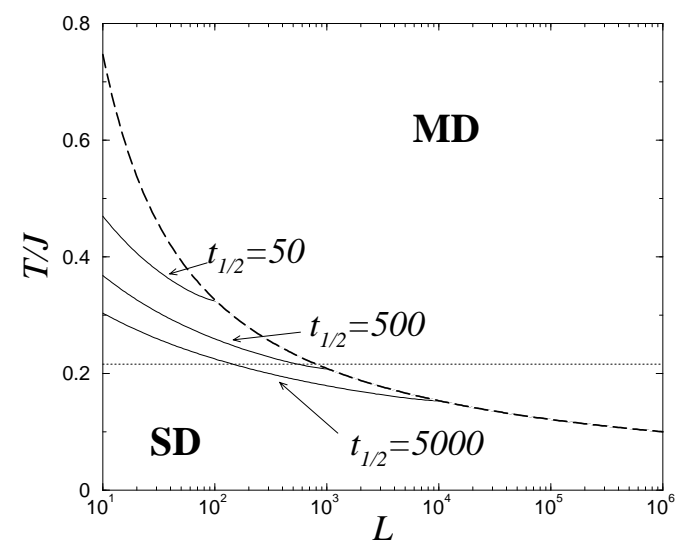

FIG. 1: Metastable decay modes and crossovers in the dynamic phase diagram for the two-dimensional kinetic Ising ferromagnet in a square-wave oscillating field with amplitude $H=2.0 \mathrm{~J}$. The dashed curve is the dynamic spinodal (DSP) $T_{\mathrm{DSP}}(L)$, separating the underlying multi-droplet (MD) from the single-droplet (SD) regime. The solid curves correspond to $T_{\times}(L)$ [Eq. (6)], the crossover to the dynamic "frozen" state for various half-periods $t_{1 / 2}$, indicated by arrows [in units of Monte Carlo steps per spin (MCSS)]. The horizontal dotted line indicates the temperature $T=0.216 \mathrm{~J}$, at which the dynamic phase transition (DPT) occurs in the large system-size limit for $t_{1 / 2}=500 \mathrm{MCSS}$. Along this horizontal line, for $L \gtrsim \mathcal{O}\left(10^{3}\right)$ the metastable lifetime is system-size independent, and its value is comparable to the fixed half-period.

500 MCSS. Near this line, the system exhibits a continuous DPT [1, 12, 14. Further lowering the temperature has no effect on the behavior of the dynamic order parameter $Q$, since the system is already locked into one of its symmetrybroken dynamic phases, where $P(Q)$ is sharply peaked near \pm 1 . From Fig. 1 it is clear that, in order to observe the DPT in a finite system for a given value of $t_{1 / 2}$, one has to employ a sufficiently large system [e.g., $L \gtrsim \mathcal{O}\left(10^{3}\right)$ for $t_{1 / 2}=500 \mathrm{MCSS}$ or $L \gtrsim \mathcal{O}\left(10^{2}\right)$ for $\left.t_{1 / 2}=50 \mathrm{MCSS}\right]$. In general, the larger $t_{1 / 2}$ is, the larger systems one needs in order to observe the $L \rightarrow \infty$ behavior. This example illustrates that it is essential to keep in mind the finite-size implications of the crossover between the MD and SD decay mechanisms.

Upon reducing the temperature for smaller $L$, the crossover from the MD to the SD regime occurs at a temperature well above that at which the infinite-system DPT would occur. In the SD regime the switching is stochastic [30]. The system will then exhibit stochastic resonance, and the order-parameter distribution $P(Q)$ will indeed possess multiple peaks, leading to a negative fourth-order cumulant [9]. Lowering the temperature still further reduces the probability that the magnetization switches during a finite number of periods, and the system becomes effectively "frozen." The crossover to this frozen phase is approximately marked by the curve along which the probability is $1 / 2$ that the magnetization does not switch during a half-period in which it is opposite to the direction of the field. This crossover curve can be estimated by noting that SD switching is brought about by the nucleation of a single droplet, which is a Poisson process. Thus, the probability that the magnetization does not change sign during a half-period in which it started off opposite to the field is

$$
P_{\text {not }}\left(t_{1 / 2} ; L, T, H\right)=\left\{\begin{array}{ll}
\exp \left\{-\left[t_{1 / 2}-t_{\mathrm{g}}(L, T, H)\right] / t_{\mathrm{n}}(L, T, H)\right\} & \text { for } t_{\mathrm{g}} \leq t_{1 / 2} \\
1 & \text { for } t_{\mathrm{g}}>t_{1 / 2}
\end{array} .\right.
$$

Setting $P_{\text {not }}=1 / 2$ leads again to an implicit equation for the corresponding crossover temperature,

$$
T_{\times}=\frac{F\left(T_{\times}, H\right)}{d \ln L+\ln \left[\frac{1}{C\left(T_{\times}, H\right) \ln 2}\left(t_{1 / 2}-\frac{L}{\left[2 \Omega_{d}\left(T_{\times}\right)\right]^{1 / d} v\left(T_{\times}, H\right)}\right)\right]} .
$$

Estimates of $T_{\times}$at $H=2.0 \mathrm{~J}$ for three different values of $t_{1 / 2}$, obtained by numerically solving Eq. (6) [36], are shown in Fig. 目 as solid curves. Since $P_{\text {not }}$ changes rapidly between zero and unity as $T$ is reduced, the specific cut-off value of $P_{\text {not }}=1 / 2$, used to define $T_{\times}$here, is not essential. For each value of $t_{1 / 2}$, the curves representing $T_{\mathrm{DSP}}$ and $T_{\times}$ form the border of a wedge-shaped region in which SR is observed (see Fig. (1). This is the regime where the dynamic order parameter is indeed characterized by a multi-peaked probability density and a negative fourth-order cumulant [9]. However, the above theoretical arguments imply that the SR behavior is a finite-size effect. We therefore conclude that the otherwise sound simulation results that Acharyya obtained in the stochastic regime [9], were misinterpreted by him as signs of a first-order DPT. 
To summarize the periodic response for the ferromagnetic Ising model, for an infinite (or sufficiently large) system, the system undergoes a continuous DPT when the the half-period becomes comparable with the metastable lifetime. Crossing the dynamic phase boundary (Fig. 11) by changing the temperature, for fixed and low frequencies (long halfperiods), the metastable lifetime becomes comparable to the half-period at appropriately low temperatures, thus the DPT occurs at a low temperature (horizontal dotted line in Fig. 11). For finite and too small systems, however, the underlying decay mode crosses over to the SD regime before the DPT occurs (crossing the dashed curve from above in Fig. 1). Then the system exhibits SR in the wedge-shape region, until practically no switching occurs during any finite observation time (frozen state). As we show next, careful analysis of simulation results for systems of different sizes reveals that the signatures attributed to a first-order DPT in Ref. [9] indeed disappear in the $L \rightarrow \infty$ limit, as predicted by the theoretical arguments presented above.

\section{SIMULATION RESULTS AND FINITE-SIZE EFFECTS}

To model spatially extended bistable systems we performed dynamic Monte Carlo simulations of a two-dimensional kinetic Ising ferromagnet below its equilibrium critical temperature. This simple model has, for example, been shown to be appropriate for describing magnetization dynamics in highly anisotropic single-domain nanoparticles and uniaxial thin films 18, 19, 20, 40]. Despite its simplicity, it is believed to capture the generic features of periodically driven, spatially extended bistable systems. The system, which is defined on a two-dimensional square lattice of linear size $L$, is described by the Hamiltonian

$$
\mathcal{H}=-J \sum_{\langle i, j\rangle} s_{i} s_{j}-H(t) \sum_{i=1}^{L^{2}} s_{i},
$$

where $s_{i}= \pm 1$ is the state of the $i$ th spin, $J>0$ is the ferromagnetic coupling constant, $\sum_{\langle i, j\rangle}$ runs over all nearestneighbor pairs, and $H(t)$ is an oscillating, spatially uniform applied field. We use a square-wave field with amplitude $H$. This has obvious computational advantages over a sinusoidal field, while it does not change the universal characteristics of the system response [14. The dynamic used is the single-spin-flip Glauber algorithm with updates at randomly chosen sites [33, 41]. At temperature $T$, each attempted spin flip from $s_{i}$ to $-s_{i}$ is accepted with probability

$$
W\left(s_{i} \rightarrow-s_{i}\right)=\frac{e^{-\Delta E_{i} / T}}{1+e^{-\Delta E_{i} / T}},
$$

where $\Delta E_{i}$ is the energy change that would result from the accepted flip. We give the temperature $T$ in energy units by setting the Boltzmann constant $k_{\mathrm{B}}=1$ in Eq. (8). For the largest system sizes $(L \geq 1024)$ we implemented a scalable massively parallel version of this dynamic [42, 43], first proposed by Lubachevsky 44 .

The dynamic order parameter [2] is the period-averaged magnetization

$$
Q=\frac{1}{2 t_{1 / 2}} \oint m(t) d t,
$$

where $m(t)=L^{-2} \sum_{i} s_{i}(t)$. The beginning of the period is chosen at a time when $H(t)$ changes sign. In particular, we compute both types of period averages, staring at the instant when $H(t)$ changes from $+H$ to $-H$, and also starting when it changes from $-H$ to $+H$. Both observations are included in the order-parameter histograms with equal weight. This simplest form of phase averaging is sufficient to produce a symmetric distribution for $Q$, in particular in the stochastic regime.

Large-scale simulations and finite-size scaling studies of the DPT have been recently performed with both sinusoidal [1, 12 and square-wave 14] fields. The results imply that the system undergoes a continuous phase transition as the half-period $t_{1 / 2}$ becomes comparable to the average metastable lifetime $\langle\tau(T, H)\rangle$. Recall that the lifetime becomes independent of the system size for large systems. The critical exponents for the dynamic order parameter and its fluctuations at the DPT are consistent with those of the two-dimensional equilibrium Ising transition 11, 12, 14, 17. In those studies, the temperature and the field amplitude were held fixed, resulting in a fixed lifetime $\langle\tau(T, H)\rangle$. The DPT was approached by tuning the half-period $t_{1 / 2}$ of the oscillating field so that it became comparable to $\langle\tau(T, H)\rangle$. An advantage of this approach is that if the smallest system is already in the MD regime, all the larger ones are, as well. Thus, one does not have to deal with subtle crossovers corresponding to the different underlying decay modes (SD vs MD). In the present study, we keep $t_{1 / 2}$ fixed and tune the metastable lifetime by varying the temperature $T$. The motivation for this is to closely parallel the study by Acharyya [9], and to show that ignoring the finite-size effects and the resulting crossovers, one can easily misinterpret the stochastic-resonance behavior in the stochastic regime as indicating a first-order transition. 

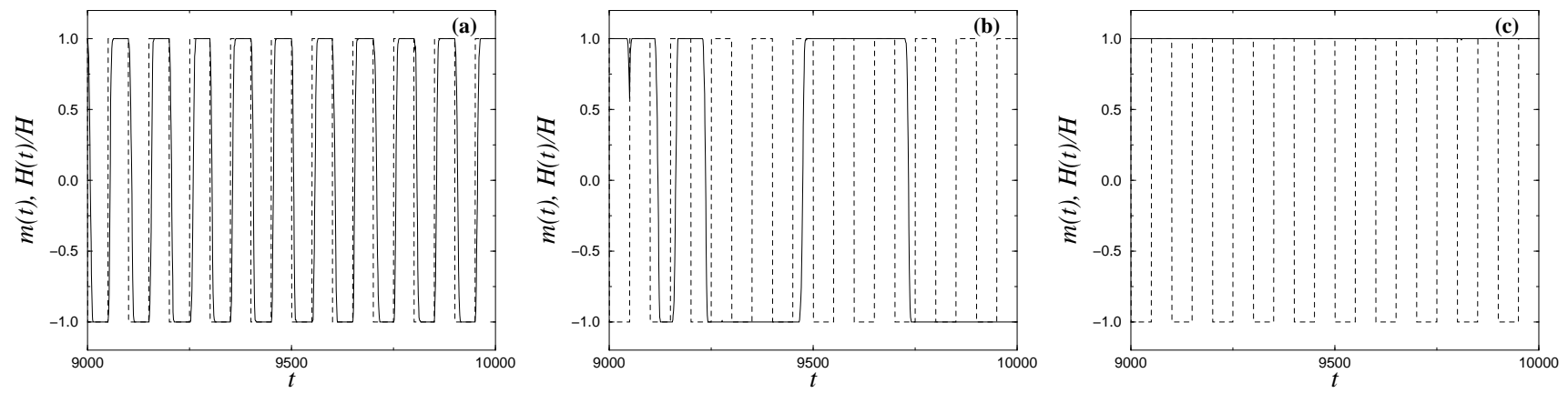

FIG. 2: Magnetization time series $m(t)$ (solid curves) and normalized applied field $H(t) / H$ (dashed curves) with $H=2.0 J$ and $t_{1 / 2}=50 \mathrm{MCSS}$, shown for a "small" system with $L=16$ at different temperatures. (a) $T=0.8 J$, dynamically disordered phase. (b) $T=0.4 J$, stochastic resonance. (c) $T=0.35 J$, dynamically "frozen" state.

Tracing the magnetization time series $m(t)$ already reflects the major qualitative differences between the responses for "small" and "large" systems, as shown in Figs. 2 and 3, respectively. For sufficiently small $L$, as the temperature is reduced, the system enters the stochastic regime, characterized by occasional random switches [Fig. 2(b)], before becoming completely "frozen" [Fig. 2(c)]. For large $L$, the system undergoes a DPT characterized by the slow wandering of the period-averaged magnetization [Fig. 3(b)] on its way to performing an asymmetric limit cycle in the dynamically ordered phase at still lower temperatures [Fig. 3(c)].

We performed simulations on system sizes ranging from $L=16$ to 2048, choosing various field amplitudes $H$ and half-periods $t_{1 / 2}$ that were kept fixed while the temperature $T$ was varied. The time unit used is one Monte Carlo step per spin [MCSS], i.e., one random "sweep" of the $L \times L$ lattice. The lengths of the runs were $10^{3}$ full periods of the oscillating field for $t_{1 / 2}=500 \mathrm{MCSS}$ and $10^{4}$ full periods for all the other values of $t_{1 / 2}$. Measuring the periodaveraged magnetization $Q$ after each half-period, we constructed averages of the norm of the order parameter, $\langle|Q|\rangle_{L}$. We further calculated the fourth-order cumulant ratio,

$$
U_{L}=1-\frac{\left\langle Q^{4}\right\rangle_{L}}{3\left\langle Q^{2}\right\rangle_{L}^{2}}
$$

which typically provides a strong indication of the nature of any underlying phase transition [33]. For a continuous transition, $U_{L}$ changes monotonically from 0 to $2 / 3$ as one tunes the system from the symmetric (disordered) to the symmetry-broken (ordered) phase. On the other hand, for a first-order transition, $U_{L}$ develops a minimum, whose location corresponds to the transition point. We also constructed histograms of $Q$, representing the order-parameter distribution $P(Q)$. In the stochastic regime, we in addition measured the residence times $t_{\mathrm{r}}$ and constructed their probability distribution $P_{\mathrm{r}}\left(t_{\mathrm{r}}\right)$, the residence-time distribution $(\mathrm{rtd})$. Here $t_{\mathrm{r}}$ is defined as the time spent in one of the two "wells" of the underlying system free energy between two consecutive switching events. It is measured as the time elapsed between two consecutive zero-crossings of $m(t)$. The residence times and their distribution are useful to characterize the system in the stochastic SD regime.

Keeping $H$ and $t_{1 / 2}$ fixed, we performed simulations measuring $\langle|Q|\rangle$ and $U_{L}$ as functions of $T$ for a series of system sizes. Here we present the results for four different pairs of values of $H$ and $t_{1 / 2}$. Figures 4 , 6, 6, and 7 show the results for $H=2.0 \mathrm{~J}$ and $t_{1 / 2}=50 \mathrm{MCSS}, H=2.0 \mathrm{~J}$ and $t_{1 / 2}=500 \mathrm{MCSS}, H=1.8 \mathrm{~J}$ and $t_{1 / 2}=20 \mathrm{MCSS}$, and for $H=3.0 \mathrm{~J}$ and $t_{1 / 2}=20 \mathrm{MCSS}$, respectively. As indicated by these figures, the findings in all these cases are qualitatively the same. For the purpose of discussion, we use Fig. 4 , corresponding to $H=2.0 \mathrm{~J}$ and $t_{1 / 2}=50 \mathrm{MCSS}$.

\section{A. Small systems}

Even for relatively small systems, $L=16-128$, at sufficiently high temperatures the underlying metastable decay mode is MD, as illustrated in the phase diagram in Fig. [4(a). Then the system magnetization $m(t)$ follows a symmetric limit cycle [see Fig. 2(a)]. Consequently, the order-parameter distribution $P(Q)$ is sharply peaked about zero [Fig 8(a)],

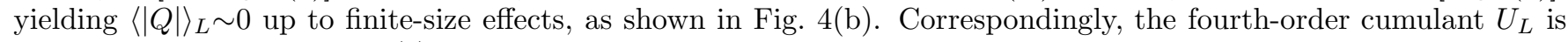
close to 0 , as shown in Fig $4(\mathrm{c})$.

As the temperature is lowered beneath $T_{\mathrm{DSP}}(L)$, the underlying decay mode crosses over to the SD regime, and the magnetization switching becomes stochastic as shown in Fig. 2(b). The order-parameter distribution then has three 

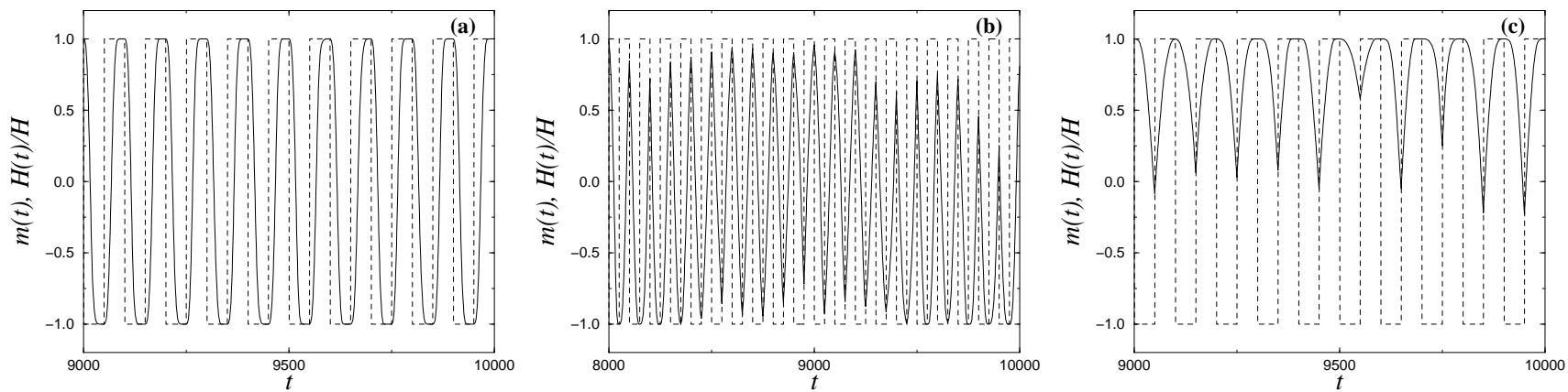

FIG. 3: Magnetization time series $m(t)$ (solid curves) and normalized applied field $H(t) / H$ (dashed curves) with $H=2.0 J$ and $t_{1 / 2}=50$ MCSS, shown for a "large" system with $L=180$ at different temperatures. (a) $T=0.5 J$, dynamically disordered phase. (b) $T=0.375 J$ near the DPT. (c) $T=0.34 J$ dynamically ordered phase.
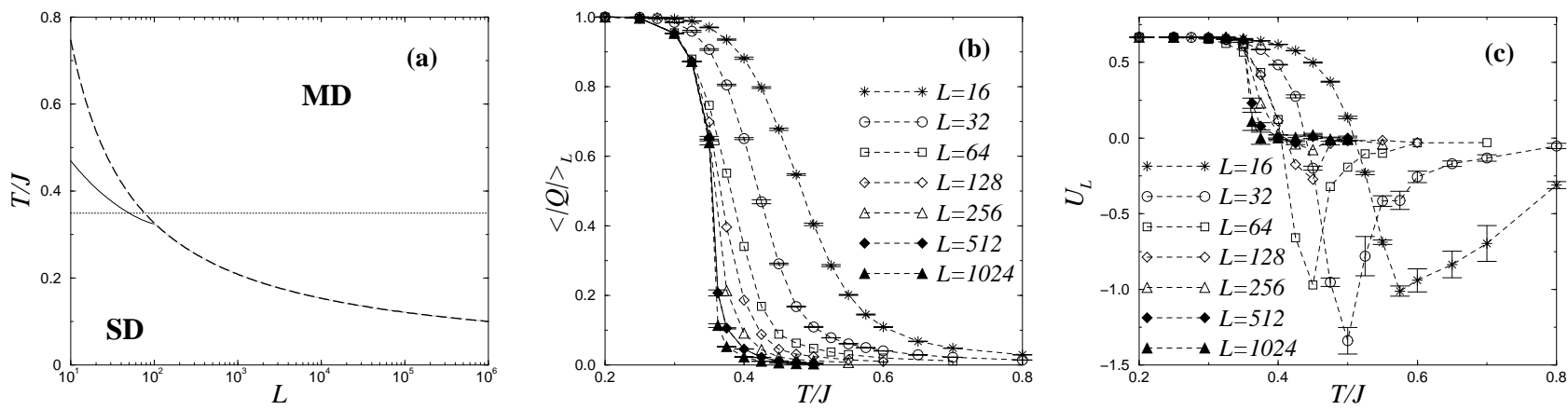

FIG. 4: Dependence of the system response on $T$ and $L$ for $H=2.0 J$ and $t_{1 / 2}=50$ MCSS. (a) Metastable dynamic phase diagram analogous to Fig. 1. The different curves have the same interpretations as in that figure. The horizontal line corresponds to $T=0.349 \mathrm{~J}$, where in the MD regime $\langle\tau(T, H)\rangle \approx t_{1 / 2}=50$ MCSS. (b) The dynamic order parameter $\langle|Q|\rangle$, shown vs $T$ for $L$ between 16 and 1024. (c) The fourth-order cumulant ratio $U_{L}$, shown vs $T$ for $L$ between 16 and 1024 . Note that the dip to negative values disappears as $L$ increases beyond 128 .
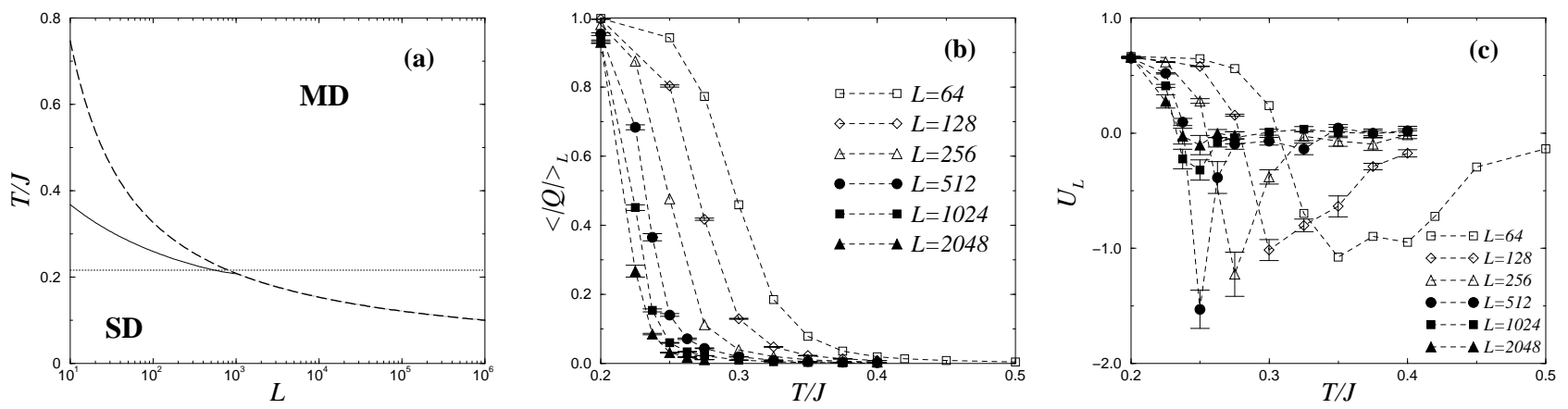

FIG. 5: Dependence of the system response on $T$ and $L$ for $H=2.0 J$ and $t_{1 / 2}=500$ MCSS. (a) Metastable dynamic phase diagram analogous to Fig. 1. The different curves have the same interpretations as in that figure. The horizontal line corresponds to $T=0.216 J$, where in the MD regime $\langle\tau(T, H)\rangle \approx t_{1 / 2}=500$ MCSS. (b) The dynamic order parameter $\langle|Q|\rangle$, shown vs $T$ for $L$ between 64 and 2048. (c) The fourth-order cumulant ratio $U_{L}$, shown vs $T$ for $L$ between 64 and 2048 . Note that the dip to negative values gradually disappears with increasing $L$. 

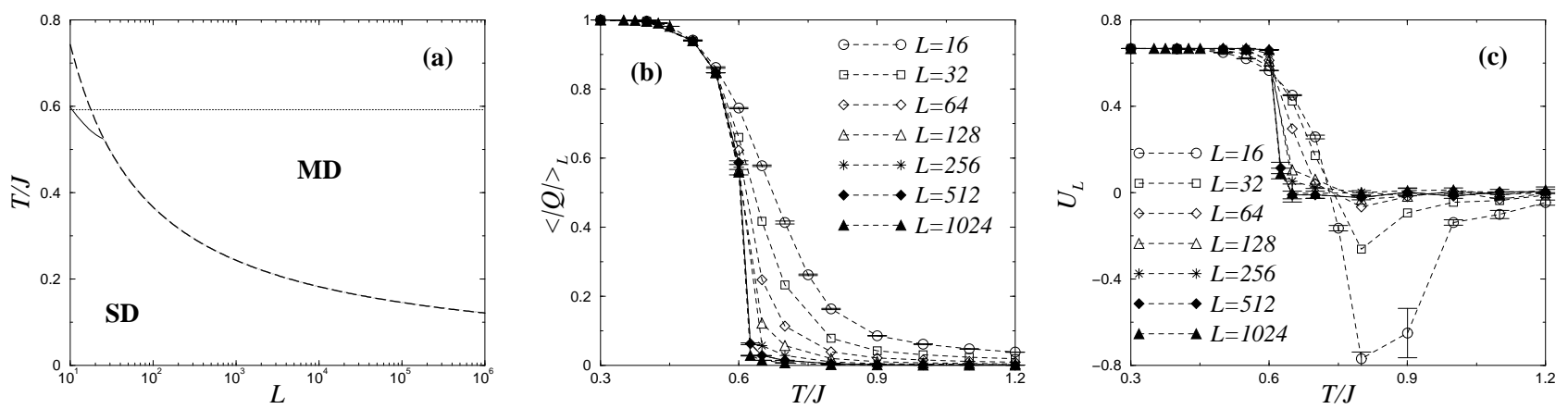

FIG. 6: Dependence of the system response on $T$ and $L$ for $H=1.8 J$ and $t_{1 / 2}=20$ MCSS. (a) Metastable dynamic phase diagram analogous to Fig. 1. The different curves have the same interpretations as in that figure. The horizontal line corresponds to $T=0.592 J$, where in the MD regime $\langle\tau(T, H)\rangle \approx t_{1 / 2}=20$ MCSS. (b) The dynamic order parameter $\langle|Q|\rangle$, shown vs $T$ for $L$ between 16 and 1024. (c) The fourth-order cumulant ratio $U_{L}$, shown vs $T$ for $L$ between 16 and 1024 . Note that the dip to negative values disappears as $L$ increases beyond 64 .
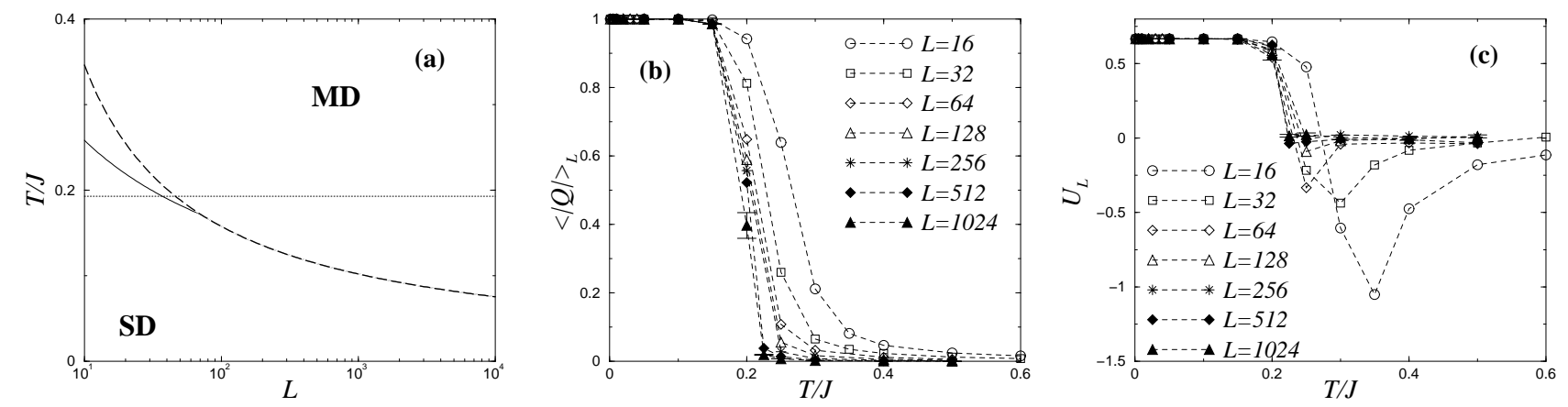

FIG. 7: Dependence of the system response on $T$ and $L$ for $H=3.0 J$ and $t_{1 / 2}=20$ MCSS. (a) Metastable dynamic phase diagram analogous to Fig. 11. The different curves have the same interpretations as in that figure. The horizontal line corresponds to $T=0.193 J$, where in the MD regime $\langle\tau(T, H)\rangle \approx t_{1 / 2}=20$ MCSS. (b) The dynamic order parameter $\langle|Q|\rangle$, shown vs $T$ for $L$ between 16 and 1024. (c) The fourth-order cumulant ratio $U_{L}$, shown vs $T$ for $L$ between 16 and 1024 . Note that the dip to negative values disappears with increasing $L$.

peaks: two extremely narrow peaks near $Q= \pm 1$ and a rather wide one centered at $Q=0$ [see Fig. 8(b) and (c)]. The peaks near \pm 1 represent the periods during which the magnetization does not switch, while the peak centered on zero represents the periods during which it switches at least once. The large width of this central peak is the result of the square-wave shape of the applied field, which results in an exponential probability density for the switching process. A sinusoidal field would yield a distribution more sharply peaked about zero, since, in that case, the switching almost always occurs when the external field assumes its maximum magnitude [9, 10, 11, 31]. The generic feature in the stochastic regime, regardless of the shape of the driving field, is the multiple-peak structure. For the square-wave field used in this paper one can obtain (see Appendix A) an analytic approximation for $P(Q)$ in the regime where $t_{1 / 2} \gg t_{g}$ [Fig. 10(a)]

$$
P(Q)=\frac{e^{-\Theta}}{2} \delta(Q+1)+\frac{\Theta}{2} e^{-\Theta|Q|}+\frac{e^{-\Theta}}{2} \delta(Q-1) .
$$

Equation (11) for $P(Q)$ is compared with simulation data in Fig. 10(b) for a system with $L=32$ at $H=2.0 \mathrm{~J}$ and $T=0.34 J$, for which $\langle\tau(T, H)\rangle_{L}=233$ MCSS. (The subscript $L$ in $\langle\tau(T, H)\rangle_{L}$ is included as a reminder that the metastable lifetime depends on $L$ in the SD regime.) The half-period is $t_{1 / 2}=500$ MCSS $\gg t_{\mathrm{g}}$. This comparison contains no fitting parameters: the average metastable lifetime of the underlying metastable decay, $\langle\tau(T, H)\rangle_{L}$, was measured in single field-reversal simulations, and its value was used to determine the scaled half-period, $\Theta=t_{1 / 2} /\langle\tau\rangle$. Instead of the delta-functions with amplitude $e^{-\Theta} / 2$, the finite value $e^{-\Theta} /(2 \Delta Q)$ was used to make the correspondence with the finite bin-size $\Delta Q$, employed to build the histogram for $Q$. In the stochastic regime the cumulant $U_{L}$ becomes negative, reaching a minimum at some temperature, as shown for the smaller values of $L$ in Fig. 1 (c). 

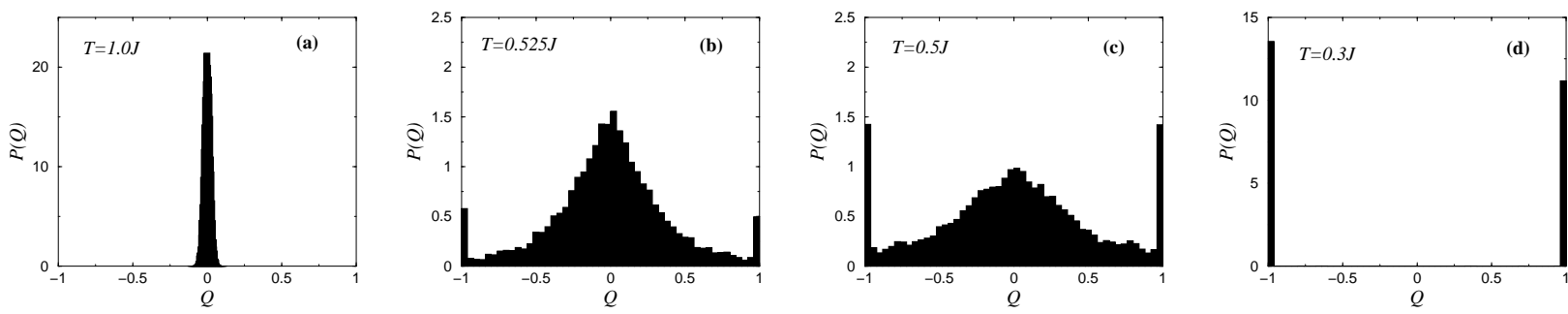

FIG. 8: Histograms representing $P(Q)$ in a small system ( $L=16$ with $H=2.0 J$ and $t_{1 / 2}=50$ MCSS) for different temperatures.
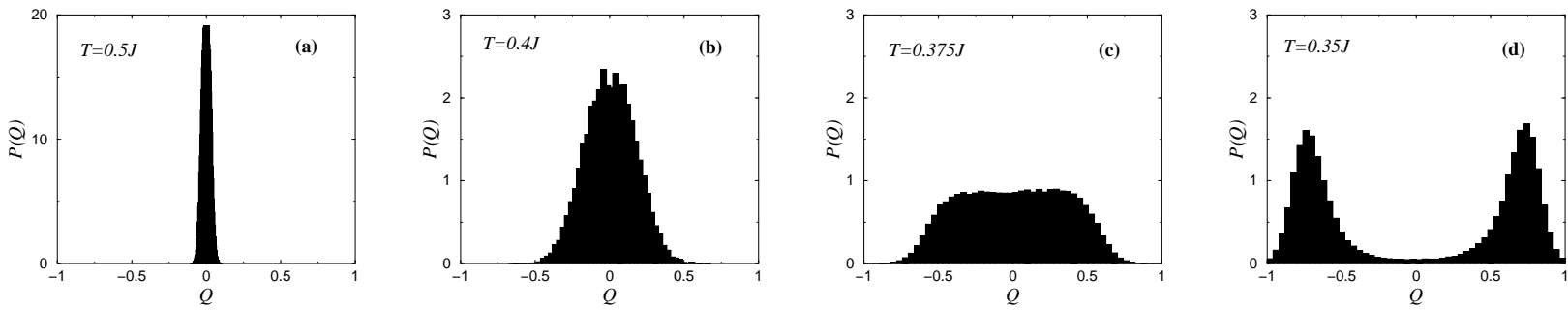

FIG. 9: Histograms representing $P(Q)$ in a large system ( $L=180$ with $H=2.0 J$ and $t_{1 / 2}=50$ MCSS) for different temperatures.

A quantity often used to detect stochastic resonance, is the $\mathrm{rtd}, P_{\mathrm{r}}\left(t_{\mathrm{r}}\right)$ [11, 28, 31, 45, 46], the probability density for the residence times $t_{\mathrm{r}}$ between zero-crossings of the magnetization. This quantity is shown in Fig. 11 for a system with $L=32$ at $H=2.0 \mathrm{~J}$ and $T=0.34 \mathrm{~J}$, for which $\langle\tau(T, H)\rangle_{L}=233 \mathrm{MCSS}$. The half-period is $t_{1 / 2}=500 \mathrm{MCSS} \gg t_{\mathrm{g}}$. The data are shown together with an analytic approximation $\left(t_{1 / 2} \gg t_{g}\right)$, which is derived in Appendix B

$$
P_{\mathrm{r}}\left(t_{\mathrm{r}}\right)=\frac{1}{\langle\tau\rangle} \frac{e^{-n \Theta}}{1-e^{-\Theta}} \times\left\{\begin{array}{ll}
\sinh \left[\frac{t_{\mathrm{r}}}{\langle\tau\rangle}-2(n-1) \Theta\right] & \text { if } 2(n-1) t_{1 / 2}<t_{\mathrm{r}}<(2 n-1) t_{1 / 2} \\
\sinh \left[2 n \Theta-\frac{t_{\mathrm{r}}}{\langle\tau\rangle}\right] & \text { if }(2 n-1) t_{1 / 2}<t_{\mathrm{r}}<2 n t_{1 / 2}
\end{array} .\right.
$$

The analytic form contains as a parameter the average lifetime $\langle\tau(T, H)\rangle_{L}$, which was measured in independent fieldreversal simulations. Thus, as for the order-parameter distribution above, no fitting parameters are involved in the comparison between the simulation data and the analytical form. The generic feature of the rtd is the structure of the peaks, which are centered at odd multiples of the half-period (i.e., at $(2 n-1) t_{1 / 2}, n=1,2, \ldots$ ), with exponentially decaying heights, as has also been observed in simulations with a sinusoidally varying field [9], 11, 31]. This behavior is characteristic of systems undergoing stochastic resonance 28, 45, 46].

In the phase diagram shown in Fig. 月(a), the weak-noise stochastic-resonance 28] behavior described here occurs in the wedge-shaped region between the two crossover curves, $T_{\mathrm{DSP}}(L)$ and $T_{\times}(L)$, as already discussed in Sec. II. Analogous behavior was discussed in detail in Ref. [31] for the case of a sinusoidal driving field.

As $T$ is lowered further for the small system, the average metastable lifetime quickly increases, and the probability of not switching during a half-period, $P_{\text {not }}\left(t_{1 / 2} ; L, T, H\right)$, approaches unity. As discussed in Sec. II, the crossover temperature $T_{\times}(L)$ corresponds to $P_{\text {not }}=1 / 2$. Consequently, switching events become rare, and the central peak in $P(Q)$ essentially disappears, leaving only the two sharp peaks near \pm 1 [see Fig. $8(\mathrm{~d})]$. At the same time, $U_{L}$ again becomes positive [see Fig. $1(\mathrm{c})$ ]. Significantly below $T_{\times}(L)$, switching will never be observed during a finite number of periods, and the system is completely "frozen" into one of its two metastable wells [see Fig. 2 $2(\mathrm{c})]$. This yields $\langle|Q|\rangle \approx 1$ and $U_{L} \approx 2 / 3$, as shown in Fig. $4(\mathrm{~b})$ and (c), respectively.

\section{B. Large systems}

For larger systems $\left[L \gtrsim \mathcal{O}\left(10^{2}\right)\right.$ for $\left.t_{1 / 2}=50 \mathrm{MCSS}\right]$, at high temperatures the system is deeply in the MD regime [Fig $\Theta(\mathrm{a})]$, where the lifetime is independent of $L$. Here, $\langle\tau(T, H)\rangle$ is significantly smaller than $t_{1 / 2}$. The limit cycle of the magnetization is symmetric [Fig. 3(a)], and the fluctuations in $Q$ are Gaussian and centered about zero [Fig. 9(a) 

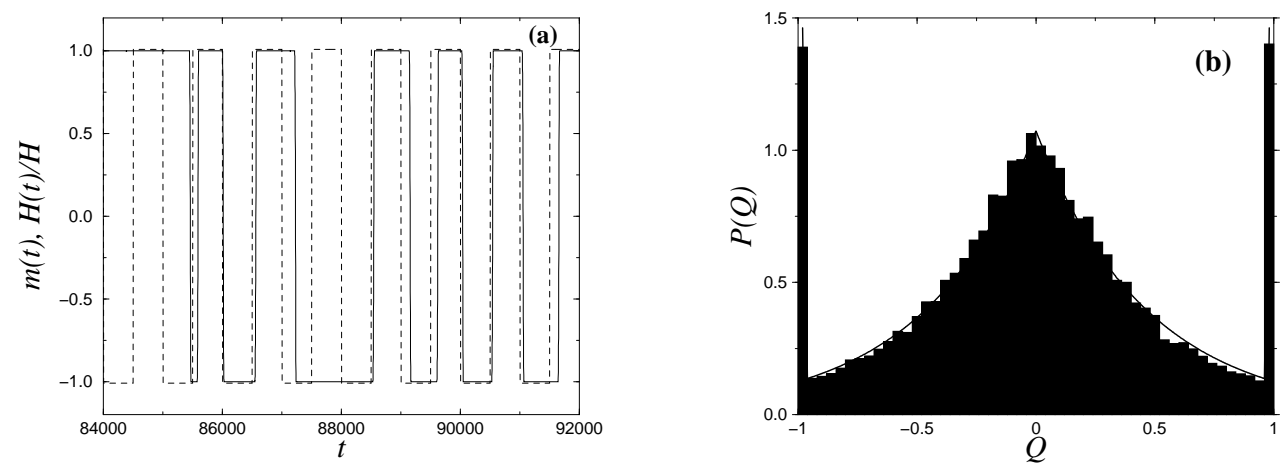

FIG. 10: Order-parameter statistics for $H=2.0 J, T=0.34 J$, and $L=32$, which yield $\langle\tau(T, H)\rangle_{L}=233$ MCSS, with $t_{1 / 2}=500 \mathrm{MCSS}$. These are the same parameters as for Fig. 11. For these parameters the system is in the stochastic regime, and $t_{\mathrm{g}}$ is negligible compared to $t_{1 / 2}$ and $\langle\tau(T, H)\rangle_{L}$. (a) A short segment of the magnetization time series. The interpretation of the line types is the same as in Fig. 2. (b) Comparison of the simulated (histogram) and the analytic [solid curve, Eq. (11)] order-parameter distributions.
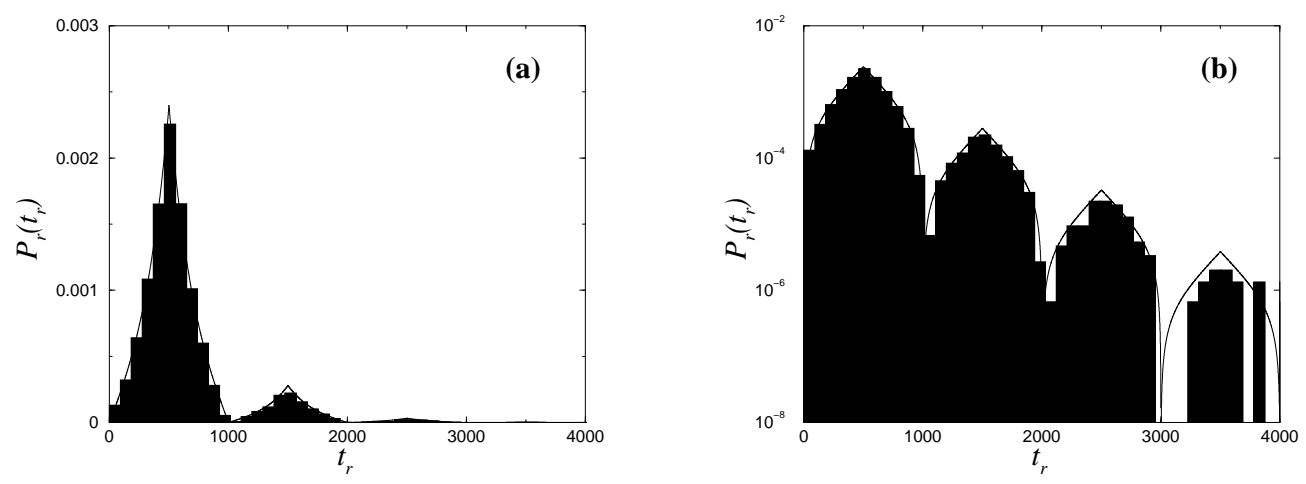

FIG. 11: Comparison of the simulated and the analytic [Eq. (12)] residence-time distributions, $P_{\mathrm{r}}\left(t_{\mathrm{r}}\right)$ in the stochastic regime where $t_{\mathrm{g}} \ll t_{1 / 2}$. Shown for a small system with $L=32$ at $H=2.0 J$ and $T=0.34 J$, where $\langle\tau(T, H)\rangle_{L}=233$ MCSS. The half-period, $t_{1 / 2}=500 \mathrm{MCSS}$, is much longer than the growth time. (a) Shown on a linear scale. (b) The same as (a), using a linear-log scale to emphasize the smaller peaks and the exponential dependence of the peak heights on $t_{\mathrm{r}}$.

and (b)]. In this regime, both $\langle|Q|\rangle_{L} \sim 0$ and $U_{L} \sim 0$, up to finite-size effects [Fig. 1 (b) and (c)]. Upon lowering the temperature, the underlying decay mode remains $\mathrm{MD}$, but the lifetime increases and eventually becomes comparable with the half-period. This happens well before $T$ reaches $T_{\mathrm{DSP}}(L)$. When $\langle\tau(T, H)\rangle$ becomes approximately equal to $t_{1 / 2}$, the system undergoes a genuine continuous phase transition, the DPT [1, 12, 14]. The system magnetization performs a slow "wandering" motion [Fig. 3(b)], and the distribution for $Q$ widens significantly [Fig. 9(c)]. Below the transition, $P(Q)$ becomes bimodal [Fig. 9(d)], $\langle|Q|\rangle_{L} \sim \mathcal{O}(1)$ [Fig. $4(\mathrm{~b})$ ], and $U_{L}$ approaches its ordered-phase value $2 / 3$ without exhibiting negative values or a minimum [Fig. A (c)]. Also, $U_{L}$ for different large values of $L$ intersect at the temperature corresponding to the DPT [Figs. G(c), E(c), 6(c), and $\mathrm{G}(\mathrm{c})]$, as expected for a continuous phase transition 33]. Detailed finite-size-scaling analysis of Monte Carlo simulations for systems that are large enough that the underlying metastable decay mode is MD, are found in Refs. 11, 12, 14.

As $T$ is reduced further, the underlying decay mode crosses over to the $\mathrm{SD}$ regime at $T_{\mathrm{DSP}}(L)$. This leads to extremely large metastable average lifetimes, such that $P_{\text {not }}$ approaches unity. However, this has no effect on the observables: below the DPT, the system is already performing asymmetric limit cycles, confined to one of its metastable wells [Fig.3(c)]. 


\section{Comparison}

From the above discussion for small and large systems, it is clear that the qualitative behavior observed as the temperature is varied depends strongly on the field amplitude, the half-period, and the system size. For example, for $H=2.0 \mathrm{~J}$ and $t_{1 / 2}=50 \mathrm{MCSS}$ one must employ $L \gtrsim \mathcal{O}\left(10^{2}\right)$ in order for the underlying metastable decay mode to become $\mathrm{MD}$, so that the DPT is observed [Fig. 4(c)]. For the same field amplitude and $t_{1 / 2}=500$ MCSS one needs $L \gtrsim \mathcal{O}\left(10^{3}\right)$ to achieve the same effect [Fig. $5(\mathrm{c})]$. For larger and larger half-periods, the "infinite"-system DPT $\left(\langle\tau(T, H)\rangle \approx t_{1 / 2}\right)$ occurs at lower and lower temperatures. However, at these low temperatures, it takes exponentially large systems to be in the MD regime, as seen from Eq. (4). By increasing $t_{1 / 2}$, one therefore quickly reaches the limit of any available computational resources.

\section{SUMMARY AND CONCLUSION}

We addressed the finite-size effects of the periodic response of spatially extended bistable systems by studying the two-dimensional kinetic Ising model in an oscillating external field. The intimate connection between the underlying metastable decay modes and the periodic response of spatially extended bistable systems has been stated and demonstrated several times [1, 10, 11, 12, 14, 31]. On the other hand, it has been claimed repeatedly [9, 26, 27] that for large enough periods (low-frequency regime), the dynamic phase transition (DPT) becomes first-order. In the present study we focused explicitly on demonstrating that any signatures resembling a first-order transition at lower temperatures are merely finite-size effects that disappear as $L$ is increased sufficiently.

First, we reviewed the basics of the well-known theory of homogeneous nucleation. Understanding the relevant timeand length scales and the various decay modes [multi-droplet (MD) and single-droplet (SD)] in metastable decay, one can estimate the important system-size dependent crossovers for the periodic response. Next we presented extensive new simulation results indicating that no first-order transition exists for any frequency, and consequently, there can be no tri-critical point separating lines of first-order and continuous dynamic phase transitions. The behavior, correctly observed in Ref. [9] but misinterpreted as indicating the existence of a first-order DPT, is due to the stochastic nature of the underlying single-droplet metastable decay. In this regime the system exhibits stochastic resonance. However, this behavior does not survive in the large-system limit with fixed field amplitude.

\section{Acknowledgments}

G.K. and M.A.N thank Z. Toroczkai for his hospitality and for using the facilities at CNLS, Los Alamos National Laboratory, where part of this manuscript was completed. We acknowledge the support of NSF through Grant Nos. DMR-9871455, DMR-9981815, DMR-0120310, and DMR-0113049, and through the support of Research Corporation Grant No. RI0761. This research used resources of the National Energy Research Scientific Computing Center, which is supported by the Office of Science of the U.S. Department of Energy under Contract No. DE-AC03-76SF00098.

\section{APPENDIX A: ANALYTIC APPROXIMATION FOR THE ORDER-PARAMETER DISTRIBUTION IN THE STOCHASTIC REGIME}

In the stochastic regime, for large $t_{1 / 2}$, we can neglect the growth time after the nucleation of the critical droplet and approximate the switching process by instantaneous switching of $m(t)$ between \pm 1 [Fig. 10. (a)]. Then, knowing that the nucleation of a critical droplet is a Poisson process (i.e., the probability density of the switching time is exponential) with rate $\langle\tau\rangle^{-1}$, we can calculate the probability density function (pdf) for the dynamic order parameter $P(Q)$. It is important to recall that in the definition of $Q$ we included both types of averaging: averaging over a period when the driving field starts with negative value and also when it starts with a positive value in the first half-period. Here we show the calculation of the former case $P_{-}(Q)$. The calculation for the latter is identical and at the end one simply has to add them together with weight $1 / 2$, resembling the way the histogram was collected: $P(Q)=\left[P_{-}(Q)+P_{+}(Q)\right] / 2$.

When the driving field is negative in the first half-period, there are five mutually distinct scenarios as illustrated on the schematic plots in Fig. 12(a-e). In cases (a), (b), and (c) the value of the magnetization $m(t)$ is +1 at the beginning of the period, while in cases $(\mathrm{d})$ and $(\mathrm{e})$ it is -1 . Since we are interested in the stationary distribution of $Q$, first we have to find the stationary probabilities $p_{\infty}^{+}\left(p_{\infty}^{-}\right)$that the magnetization has the value $+1(-1)$ at the beginning of a period. After a quick look at the five cases above [Fig. 12(a-e)], one can write down a set of 

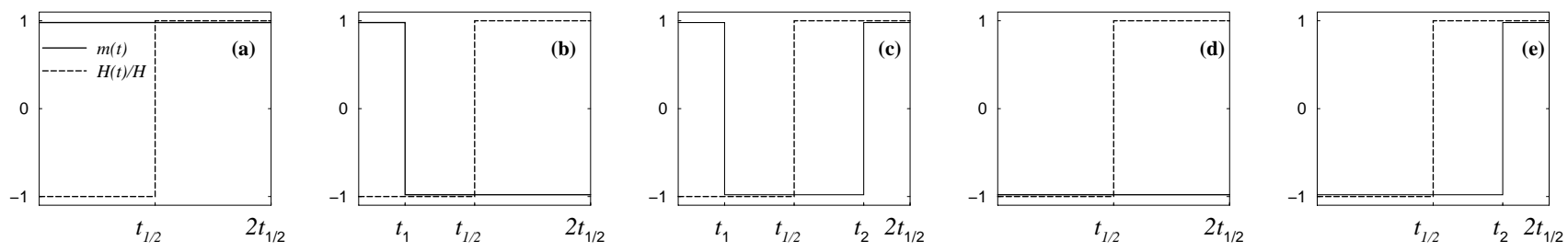

FIG. 12: Schematic plots for constructing the probability density $P_{-}(Q)$ when the driving field is negative in the first halfperiod. Dashed and solid lines represent the driving field and the magnetization, respectively. The switching times $t_{1}$ and $t_{2}$, possibly occurring in the first and second half-period, respectively, are measured from the beginning of their respective half-periods.

discrete-time "time-evolution" equations (from one period to the next)

$$
\begin{aligned}
& p_{n+1}^{+}=\left[e^{-\Theta}+\left(1-e^{-\Theta}\right)^{2}\right] p_{n}^{+}+\left(1-e^{-\Theta}\right) p_{n}^{-} \\
& p_{n+1}^{-}=\left(1-e^{-\Theta}\right) e^{-\Theta} p_{n}^{+}+e^{-\Theta} p_{n}^{-}
\end{aligned}
$$

for $p_{n}^{+}$and $p_{n}^{-}$, the probabilities that the magnetization is +1 and -1 at the beginning of the $n$th period, respectively. In Eq. (A1) we used the definition $\Theta=t_{1 / 2} /\langle\tau\rangle$. From these equations one can easily obtain the stationary-state (fixed-point) values $p_{\infty}^{+}=1 /\left(1+e^{-\Theta}\right)$ and $p_{\infty}^{-}=e^{-\Theta} /\left(1+e^{-\Theta}\right)$. Now for each case (a-e), we consider the conditional probability density of $Q$ (conditional on the value of the magnetization at the beginning of the period). In case (a) $Q=1$ with probability $e^{-\Theta}$, i.e., that the magnetization does not switch in the first half-period, yielding a deltafunction contribution $e^{-\Theta} \delta(Q-1)$ to the full pdf. In case (b) the magnetization switches at $t_{1}$ in the first halfperiod and does not switch back in the second, resulting in $Q=\left(t_{1}-t_{1 / 2}\right) / t_{1 / 2}$. The contribution to the pdf is $e^{-\Theta}\left\langle\delta\left(Q-\left(t_{1}-t_{1 / 2}\right) / t_{1 / 2}\right)\right\rangle_{t_{1}}$, where $\langle\ldots\rangle_{t_{1}}$ is an averaging over the exponentially distributed switching time $t_{1}$. In case (c) the magnetization switches twice, at $t_{1}$ in the first half-period and at $t_{2}$ in the second one, resulting in $Q=\left(t_{1}-t_{2}\right) / t_{1 / 2}$. The contribution to the pdf is $\left\langle\delta\left(Q-\left(t_{1}-t_{2}\right) / t_{1 / 2}\right)\right\rangle_{t_{1}, t_{2}}$, where $\langle\ldots\rangle_{t_{1}, t_{2}}$ is an averaging over the exponentially distributed switching times $t_{1}$ and $t_{2}$, occurring independently in the first and second half-period (and measured from the beginning of their respective half-periods). In case (d) the initial value of the magnetization is -1 and it does not switch in the second half-period, yielding $e^{-\Theta} \delta(Q+1)$. In case (e) the magnetization switches once at $t_{2}$ in the second half-period resulting in $Q=-t_{2} / t_{1 / 2}$ and a contribution $\left\langle\delta\left(Q+t_{2} / t_{1 / 2}\right)\right\rangle_{t_{2}}$ to the pdf. Combining the above conditional pdfs with the probabilities of the corresponding initial values of magnetization, one obtains

$$
\begin{aligned}
P_{-}(Q)= & p_{\infty}^{+}\left\{e^{-\Theta} \delta(Q-1)+e^{-\Theta}\left\langle\delta\left(Q-\left(t_{1}-t_{1 / 2}\right) / t_{1 / 2}\right)\right\rangle_{t_{1}}+\left\langle\delta\left(Q-\left(t_{1}-t_{2}\right) / t_{1 / 2}\right)\right\rangle_{t_{1}, t_{2}}\right\} \\
& +p_{\infty}^{-}\left\{e^{-\Theta} \delta(Q+1)+\left\langle\delta\left(Q+t_{2} / t_{1 / 2}\right)\right\rangle_{t_{2}}\right\} .
\end{aligned}
$$

Carrying out the averages above using the exponential pdfs for $t_{1}$ and $t_{2}$ we arrive at

$$
\begin{aligned}
P_{-}(Q)= & \frac{1}{1+e^{-\Theta}}\left\{e^{-\Theta} \delta(Q-1)+H(-Q) \Theta e^{-\Theta(2-|Q|)}+\frac{\Theta}{2}\left(e^{-\Theta|Q|}-e^{-\Theta(2-|Q|)}\right)\right\} \\
& +\frac{e^{-\Theta}}{1+e^{-\Theta}}\left\{e^{-\Theta} \delta(Q+1)+H(-Q) \Theta e^{-\Theta|Q|}\right\},
\end{aligned}
$$

where $H(x)$ is the Heaviside step-function. An identical calculation for the pdf of $Q$ for periods starting with positive value of the driving field yields

$$
\begin{aligned}
P_{+}(Q)= & \frac{e^{-\Theta}}{1+e^{-\Theta}}\left\{e^{-\Theta} \delta(Q-1)+H(Q) \Theta e^{-\Theta|Q|}\right\} \\
& +\frac{1}{1+e^{-\Theta}}\left\{e^{-\Theta} \delta(Q+1)+H(Q) \Theta e^{-\Theta(2-|Q|)}+\frac{\Theta}{2}\left(e^{-\Theta|Q|}-e^{-\Theta(2-|Q|)}\right)\right\} .
\end{aligned}
$$

The symmetrized ("phase-averaged") dynamic order-parameter becomes considerably simpler and easier to compare with measured histograms

$$
P(Q)=\frac{1}{2}\left[P_{+}(Q)+P_{-}(Q)\right]=\frac{e^{-\Theta}}{2} \delta(Q+1)+\frac{\Theta}{2} e^{-\Theta|Q|}+\frac{e^{-\Theta}}{2} \delta(Q-1) .
$$




\section{APPENDIX B: RESIDENCE-TIME DISTRIBUTION AND ITS ANALYTIC APPROXIMATION IN THE STOCHASTIC REGIME}

In the stochastic-resonance limit, where $\langle\tau\rangle$ is not much smaller than $t_{1 / 2}$, while both are much larger than $t_{\mathrm{g}}$, we can obtain an analytic form for the rtd $P_{\mathrm{r}}\left(t_{\mathrm{r}}\right)$. The derivation follows that given for a sinusoidally oscillating field in the Appendix of Ref. [31]. However, the present case is simpler since the probability that a switching event has not occurred within a time $t$ after the field has changed its sign to become opposite to the magnetization direction, $P_{\text {not }}(t ; L, T, H)$, is a simple exponential, $\exp \left(-t /\langle\tau(T, H)\rangle_{L}\right)$ [see Eq. (5)]. As a consequence, all the integrals that have to be evaluated numerically in the sinusoidal case, can here be trivially calculated analytically. Provided the magnetization switched in a period (say period $n=1$ ) at time $t_{1}$ (measured from the instant the driving field changed sign), the next magnetization switching occurring in the $n$th period at $t_{2}$ (also measured from the instant the driving field changed sign) results in a residence time $t_{\mathrm{r}}=(2 n-1) t_{1 / 2}-t_{1}+t_{2}$, where $t_{1}$ and $t_{2}$ are exponentially distributed variables. The formal expression for the rtd then can be written as

$$
P_{\mathrm{r}}\left(t_{\mathrm{r}}\right)=\sum_{n=1}^{\infty} \frac{e^{-(n-1) \Theta}}{1-e^{-\Theta}}\left\langle\delta\left(t_{\mathrm{r}}-(2 n-1) t_{1 / 2}+t_{1}-t_{2}\right)\right\rangle_{t_{1}, t_{2}}
$$

Carrying out the averages above yields, after some rearrangement,

$$
P_{\mathrm{r}}\left(t_{\mathrm{r}}\right)=\frac{1}{\langle\tau\rangle} \frac{e^{-n \Theta}}{1-e^{-\Theta}} \times\left\{\begin{array}{ll}
\sinh \left[\frac{t_{\mathrm{r}}}{\langle\tau\rangle}-2(n-1) \Theta\right] & \text { if } 2(n-1) t_{1 / 2}<t_{\mathrm{r}}<(2 n-1) t_{1 / 2} \\
\sinh \left[2 n \Theta-\frac{t_{\mathrm{r}}}{\langle\tau\rangle}\right] & \text { if }(2 n-1) t_{1 / 2}<t_{\mathrm{r}}<2 n t_{1 / 2}
\end{array},\right.
$$

where $n=1,2, \ldots$

[1] S. W. Sides, P. A. Rikvold, and M. A. Novotny, Phys. Rev. E 59, 2710 (1999) and references therein.

[2] T. Tomé and M. J. de Oliveira, Phys. Rev. A 41, 4251 (1990).

[3] J. F. F. Mendes and J. S. Lage, J. Stat. Phys. 64, 653 (1991).

[4] W. S. Lo and R. A. Pelcovits, Phys. Rev. A 42, 7471 (1990).

[5] M. Acharyya and B.K. Chakrabarti, Phys. Rev. B 52, 6550 (1995).

[6] M. Acharyya, Phys. Rev. E 56, 1234 (1997).

[7] M. Acharyya, Phys. Rev. E 56, 2407 (1997).

[8] M. Acharyya, Phys. Rev. E 58, 179 (1998).

[9] M. Acharyya, Phys. Rev. E 59, 218 (1999).

[10] S. W. Sides, R. A. Ramos, P. A. Rikvold, and M. A. Novotny, J. Appl. Phys. 79, 6482 (1996).

[11] S. W. Sides, R. A. Ramos, P. A. Rikvold, and M. A. Novotny, J. Appl. Phys. 81, 5597 (1997).

[12] S. W. Sides, P. A. Rikvold, and M. A. Novotny, Phys. Rev. Lett. 81834 (1998).

[13] G. M. Buendía and E. Machado, Phys. Rev. B 61, 14686 (2000).

[14] G. Korniss, C. J. White, P. A. Rikvold, and M. A. Novotny, Phys. Rev. E 63, 016120 (2001).

[15] G. M. Buendía and E. Machado, Phys. Rev. E 58, 1260 (1998).

[16] M. F. Zimmer, Phys. Rev. E 47, 3950 (1993).

[17] H. Fujisaka, H. Tutu, and P. A. Rikvold, Phys. Rev. E 63, 036109 (2001); Erratum, Phys. Rev. E 63059903 (2001).

[18] Y.L. He and G.-C. Wang, Phys. Rev. Lett. 70, 2336 (1993).

[19] Q. Jiang, H.-N. Yang, and G.-C. Wang, Phys. Rev. B 52, 14911, (1995); J. Appl. Phys. 79, 5122 (1996).

[20] J. S. Suen and J. L. Erskine, Phys. Rev. Lett. 783567 (1997); J. S. Suen, M. H. Lee, G. Teeter, and J. L. Erskine, Phys. Rev. B 59, 4249 (1999).

[21] G. S. Jeon, H. J. Kim, M. Y. Choi, B. J. Kim, and P. Minnhagen, Phys. Rev. B 65184510 (2002).

[22] H. Jang and M. J. Grimson, Phys. Rev. E 63, 066119 (2001); H. Jang, M. J. Grimson, and C. K. Hall, e-print condmat/0205070

[23] G. Grinstein, C. Jayaprakash, and Y. He, Phys. Rev. Lett. 55, 2527 (1985).

[24] K. E. Bassler and B. Schmittmann, Phys. Rev. Lett. 73, 3343 (1994).

[25] The direct correspondence between the DPT and other non-equilibrium phase transitions considered by Refs. 23, 24, is somewhat less obvious in that no microscopic dynamics is defined here for the dynamic order parameter (the periodaveraged magnetization). Further, a priori it could not be known whether or not the period-averaging in time introduces long-range effective interactions for the local dynamic order parameter.

[26] M. Acharyya and B. K. Chakrabarti in Annual Reviews of Computational Physics I, edited by D. Stauffer (World Scientific, Singapore, 1994), p. 107.

[27] B. Chakrabarti and M. Acharyya, Rev. Mod. Phys. 71, 847 (1999).

[28] L. Gammaitoni, P. Hänggi, P. Jung, and F. Marchesoni, Rev. Mod. Phys. 70, 223 (1998). 
[29] H. Tomita and S. Miyashita, Phys. Rev. B 46, 8886 (1992).

[30] P. A. Rikvold, H. Tomita, S. Miyashita, and S. W. Sides, Phys. Rev. E 49, 5080 (1994); H. L. Richards, S. W. Sides, M. A. Novotny, and P. A. Rikvold, J. Magn. Magn. Mater. 150, 37 (1995); P. A. Rikvold and B. M. Gorman, in Annual Reviews of Computational Physics I, edited by D. Stauffer (World Scientific, Singapore, 1994), p. 149.

[31] S. W. Sides, P. A. Rikvold, and M. A. Novotny, Phys. Rev. E 57, 6512 (1998).

[32] G. Korniss, P. A. Rikvold, and M. A. Novotny, in Computer Simulation Studies in Condensed Matter Physics XIV, edited by D. P. Landau, S. P. Lewis, and H.-B. Schüttler, Springer Proceedings in Physics Vol. 89, (Springer, Berlin, 2002), in press.

[33] K. Binder and D. W. Heermann, Monte Carlo Simulation in Statistical Physics. An Introduction, 3rd edn. (Springer, Berlin, 1997).

[34] K. Binder, in Finite-Size Scaling and Numerical Simulation of Statistical Systems, edited by V. Privman (World Scientific, Singapore, 1990), p. 173.

[35] N. Goldenfeld, Lectures on the Phase Transition and the Renormalization Group (Perseus Publishing, Reading, MA, 1992).

[36] In our numerical solution of Eqs. (A) and (6) we employed the low-temperature approximation for the classical theory of nucleation and growth where the shape-, prefactor, and the free energy of the critical droplet are independent of the temperature 38. In particular, for the shape factor we used $\Omega_{2} \approx 4$, corresponding to a square droplet. For the the prefactor we used $C(T, H) \approx 3 / 8,1.36$, and $5 / 4$ for $H \in(J, 2 J), H=2 J$, and $H \in(2 J, 4 J)$, respectively [38]. For the free energy of the critical droplet we used $F(T, H) \approx 16 J-6 H, 4 J$, and $8 J-2 H$ for $H \in(J, 2 J], H=2 J$, and $H \in[2 J, 4 J)$, respectively [38]. The velocity $v(T, H)$ was obtained from Eqs. (13) and (21) of Ref. [37]

[37] P. A. Rikvold and M. Kolesik, J. Stat. Phys. 100377 (2000).

[38] M. A. Novotny, in Computer Simulation Studies in Condensed Matter Physics IX, edited by D. P. Landau, K. K. Mon, and H.-B. Schüttler (Springer, Berlin, 1997); M. A. Novotny, in Computer Simulation Studies in Condensed Matter Physics $X V$, edited by D. P. Landau, S. P. Lewis, and H.-B. Schüttler (Springer, Berlin, in press), e-print cond-mat/0204098.

[39] A. Bovier and F. Manzo, e-print cond-mat/0107376 (2001).

[40] P. A. Rikvold, M. A. Novotny, M. Kolesik, and H. L. Richards, in Dynamical Properties of Unconventional Magnetic Systems, edited by A. T. Skjeltorp and D. Sherrington (Kluwer, Dordrecht, 1998), p. 307; M. Kolesik, M. A. Novotny, and P. A. Rikvold, Phys. Rev. B 56, 11791 (1997).

[41] R. J. Glauber, J. Math. Phys. 4, 294 (1963).

[42] G. Korniss, M. A. Novotny, and P. A. Rikvold, J. Comput. Phys. 153, 488 (1999).

[43] G. Korniss, Z. Toroczkai, M. A. Novotny, and P. A. Rikvold, Phys. Rev. Lett. 84, 1351 (2000).

[44] B. D. Lubachevsky, Complex Systems 1, 1099 (1987); J. Comput. Phys. 75, 10 (1996).

[45] P. Jung, Phys. Rep. 234, 175 (1993).

[46] L. Gammaitoni, F. Marchesoni, and S. Santucci, Phys. Rev. Lett. 74, 1052 (1995). 